Classification

Physics Abstracts

52.35

\title{
Experimental evidence for various mechanisms for suprathermal electron generation in P-polarized laser produced plasmas
}

\author{
G. Bonnaud (*) and E. Fabre \\ GRECO Interaction Laser Matière, CNRS, Laboratoire de Physique des Milieux Ionisés, \\ Ecole Polytechnique, 91128 Palaiseau Cedex, France
}

(Reçu le 27 septembre 1984, accepté le 22 février 1985)

\begin{abstract}
Résumé. - La dépendance angulaire de l'énergie laser absorbée dans les interactions laser-plasma, par les électrons suprathermiques a été étudiée en polarisation P. Deux longueurs d'onde de laser ont été utilisées : $10,6 \mu \mathrm{m}$ et $0,53 \mu \mathrm{m}$ avec les éclairements respectifs $10^{12}$ et $10^{14} \mathrm{~W} / \mathrm{cm}^{2}$ sur des cibles planes de terphane. Les résultats prouvent qu'en dehors de l'absorption résonnante un mécanisme crée des électrons rapides (pour un angle d'incidence du faisceau laser de $60^{\circ}$ ). Ces résultats plaident en faveur du déclenchement de l'instabilité de décomposition en deux ondes plasma.
\end{abstract}

\begin{abstract}
The angular dependence of the absorbed laser energy which appears in suprathermal electrons and their temperature have been studied in P-polarization, in laser produced plasmas. We have used two laser wavelengths $10.6 \mu \mathrm{m}$ and $0.53 \mu \mathrm{m}$ at irradiances of $10^{12}$ and $10^{14} \mathrm{~W} / \mathrm{cm}^{2}$ respectively, focused on plane mylar targets. The results show that in addition to resonant absorption fast electrons are generated (at an incidence angle of the laser beam of $60^{\circ}$ ) by another mechanism. The results are consistent with the latter, that is, with two plasmon decay instability.
\end{abstract}

\section{Introduction.}

The generation of suprathermal electrons in laser inertial fusion experiments can be a serious problem for the behaviour and efficiency of the compression. Two dangerous instabilities capable of generating fast electrons are two plasmon decay (TPD) and stimulated Raman scattering(SRS). TPD is characterized by the decay of an electromagnetic (EM) wave into two electron plasma waves and is localized at one quarter of the critical density $n_{\mathrm{c}}$. SRS, in which an EM wave decays into another EM wave and an electron plasma wave may exist for all the electron densities equal to or below $n_{\mathrm{c}} / 4$.

These two instabilities may be absolute and their thresholds in inhomogeneous plasma irradiated by a normally incident laser wave have been determined by linear fluid theory [1] :

$$
I^{\mathrm{TPD}}=6 \times 10^{15} \frac{T_{\mathrm{e}}}{\lambda_{0} L} \quad I^{\mathrm{SRS}}=\frac{T_{\mathrm{e}}}{\lambda_{0}^{2 / 3} L^{4 / 3}}
$$

(*) New address : C.E.A., Centre d'Etude de Limeil, B.P. 27, 94190 Villeneuve-St-Georges, France. 
where $T_{\mathrm{e}}$ is the electron temperature in $\mathrm{keV}, \lambda_{0}$ the laser wavelength in $\mu \mathrm{m}$ and $L$ the density scale length in $\mu \mathrm{m}$, near $n_{\mathrm{c}} / 4$; the unit of intensity if $\mathrm{W} / \mathrm{cm}^{2}$. Because of the higher threshold value and weaker saturation level [2] for the SRS, TPD dominates in general and is most efficient for fast electron generation [3].

To determine the contribution of an instability to the plasma heating, it is necessary to isolate it from other mechanisms. Laser irradiation of a thick target at various angles of incidence $\theta$ allows us to scan regions of various densities, $n_{\mathrm{e}}=n_{\mathrm{c}} \cos ^{2} \theta$, where the laser energy deposition occurs (the large focal spots allow us to assume the expansion to have planar geometry). As an example, for $\theta$ above $60^{\circ}$, the laser wave does not reach $n_{\mathrm{c}} / 4$ and then TPD cannot occur. In the case of resonant absorption an optimum is usually found for a smaller angle of incidence, in the range of $20^{\circ}$ to $30^{\circ}$.

\section{Experimental procedure.}

Our experiments have been conducted at two different laser wavelengths $10.6 \mu \mathrm{m}$ and $0.53 \mu \mathrm{m}$. The target was a 50- $\mu \mathrm{m}$ thick mylar foil. In a first set, we used a $\mathrm{CO}_{2}$ laser giving 8 joules in a $1 \mathrm{~ns}$ pulse at $10.6 \mu \mathrm{m}$. A second set was made using $17 \mathrm{~J}-200$ ps pulse at $0.52 \mu \mathrm{m}$ wavelength obtained by frequency doubling of Nd glass laser [4] emission with KDP crystal. For all these experiments, the polarization was in the plane of incidence.

The diagnostics used were : continuum X-ray emission, ion charge emission and, for experiments at $0.53 \mu \mathrm{m}$, the $3 / 2 \omega_{0}$ harmonic emission of the fundamental angular frequency of the laser $\left(\omega_{0}\right)$. The electron temperature was determined, as usual, from the spectral intensity distribution of Bremsstrahlung, using the absorber foil method. The spectrometer covered an energy range of $1-70 \mathrm{keV}$ with ten channels.

The angle of incidence was changed by rotating the target. The spectrometer was kept in a fixed direction with respect to the laser beam. Isotropy of the X-rays was found for our laser irradiances, at least in the target rotation plane. Many authors [5] have noticed the isotropy, for irradiances comparable to ours. So we could assume that the variation of X-ray emission did not originate from the detection geometry.

\section{Experiment at $10.6 \mu \mathrm{m}$.}

In order to have a properly defined angle of incidence we used a $7^{\circ}$-aperture focusing system. The focal spot diameter was $(300 \pm 50) \mu \mathrm{m}$ resulting in an irradiance of $(4 \pm 1) .10^{12} \mathrm{~W} / \mathrm{cm}^{2}$.

Figure 1 gives the variations of the signals detected on the different channels as a function of the angle of incidence of the laser beam on the target. The error bars are associated with the average over ten shots. Between 1 and $10 \mathrm{keV}$, we only noticed a monotonic decrease in the X-ray signal when $\theta$ was increasing, but the highest energy channels revealed significant changes : the signals showed a first maximum for an angle of incidence about $25^{\circ}$, and a second maximum around $60^{\circ}$.

The spectral distribution showed the usual two-temperature spectra. The low temperature presented no clear variation and its value was about $350 \mathrm{eV}$. The high temperature (Fig. 2) was almost constant between $0^{\circ}$ and $50^{\circ}$ with a weak maximum around $25^{\circ}$, giving a $6 \mathrm{keV}$ temperature. A sharp rise occurred at $60^{\circ}$. Here, the temperature was $12 \mathrm{keV}$. The energy contained in the fast electrons (Fig. 3) was deduced from the spectra using the method of Brueckner [6]. For an angle of incidence between $0^{\circ}$ and $25^{\circ}, 20 \%$ of the incident energy was found in fast electrons; at $60^{\circ}$, the fraction was below $1 \%$.

The existence of a maximum around $25^{\circ}$ must be attributed to resonant absorption. From the expression $\left(2 \pi L / \lambda_{0}\right)^{1 / 3} \sin \theta=0.8$, we deduced the density scale length, $L=(10-20) \mu \mathrm{m}$, in accordance with the absorption measurement under the same experimental conditions [7]. The large amount of energy in fast electrons for normal incidence can be explained by the existence of resonant absorption on a rippled critical layer [8] or by the occurrence of other absorption 

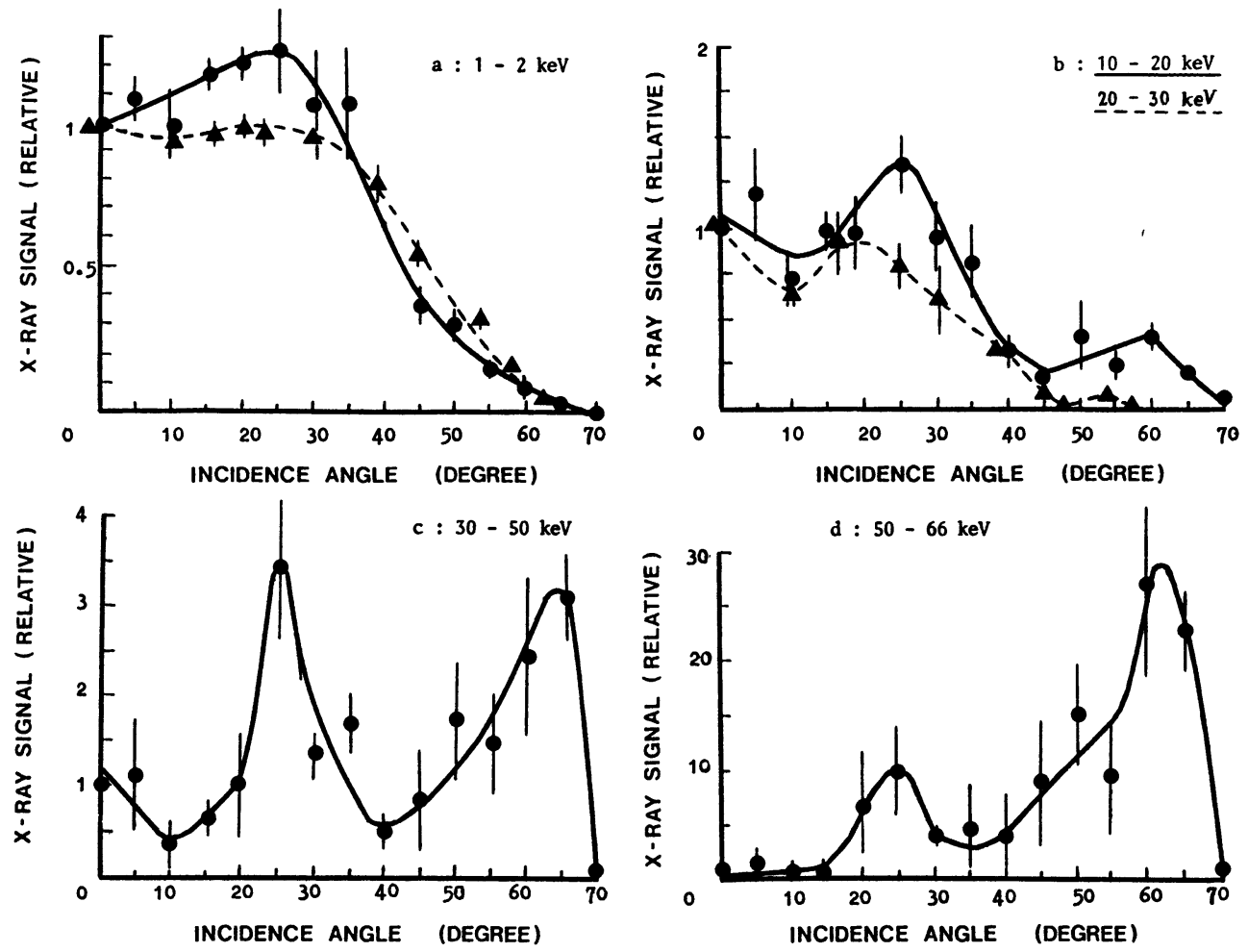

Fig. 1. - Signals of different X-ray channels versus angle of incidence for various energies of X-rays. A $50 \mu \mathrm{m}$ thick mylar plane target is irradiated with a P-polarized laser beam. Dots and solid lines correspond to a $10.6 \mu \mathrm{m}$ laser wavelength and an irradiance of $4 \times 10^{12} \mathrm{~W} / \mathrm{cm}^{2}$ at normal incidence in a $300 \mu \mathrm{m}$ focal diameter. Triangles and dashed lines have $\lambda_{0}=0.53 \mu \mathrm{m}$ and an irradiance of $2 \times 10^{14} \mathrm{~W} / \mathrm{cm}^{2}$ for $\theta=0^{\circ}$ in a $200 \mu \mathrm{m}$ focal diameter.

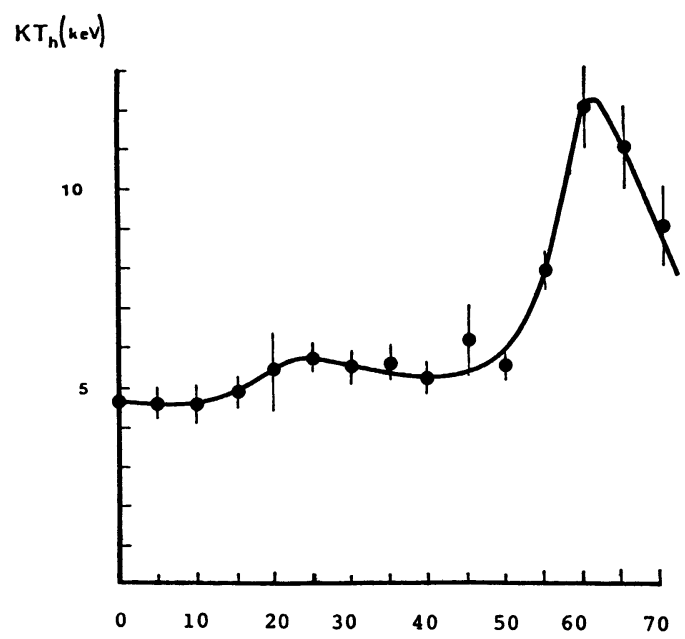

Fig. 2. - High temperature versus angle for a $50 \mu \mathrm{m}$ thick mylar plane target irradiated with a P-polarized laser; the focal diameter is $300 \mu \mathrm{m}$ and the irradiance is $4 \times 10^{12} \mathrm{~W} / \mathrm{cm}^{2}$ at normal incidence. 


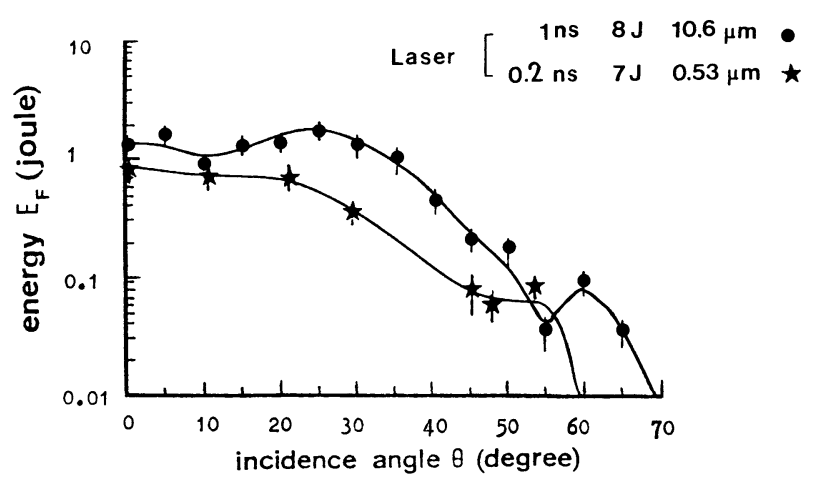

Fig. 3. - Energy of fast electrons obtained by X-ray spectra versus the angle of incidence for two different irradiation conditions : focal diameter $\varnothing=300 \mu \mathrm{m}$, irradiance $I_{0}=4 \times 10^{12} \mathrm{~W} / \mathrm{cm}^{2}$ (dots) $\varnothing \varnothing=200 \mu \mathrm{m}$, $I_{0}=2 \times 10^{14} \mathrm{~W} / \mathrm{cm}^{2}$ (stars). The laser beams are P-polarized. Laser beams impinge on a $50 \mu \mathrm{m}$ thick plane mylar target.

mechanisms, for example the decay instability. At $\theta=60^{\circ}$, the laser beam reaches the quarter critical density with a grazing incidence. Now, during its propagation in the plasma, the laser field increases gradually and reaches its maximum value at the density layer where reflection occurs. In our experimental conditions, we found a swelling factor [9] of 2 at $60^{\circ}$. So the irradiance obtained at the $n_{\mathrm{c}} / 4$ layer is higher at $60^{\circ}$ than at $0^{\circ}$. TPD is therefore favoured at a $60^{\circ}$ angle, since the threshold levels are minimal at $0^{\circ}$ and $60^{\circ}[10]$ (here threshold $\simeq 10^{12} \mathrm{~W} / \mathrm{cm}^{2}$ ) taking the density scalelength in the case of self similar expansion model. At the same time, increasing $\theta$ from 20 to $60^{\circ}$ reduces the efficiency of resonant absorption and consequently we gradually change the relative efficiencies of absorption mechanisms, favouring TPD for incidence of $60^{\circ}$. Resonant absorption and TPD are two independent processes which both generate fast electrons and also heat them to different temperatures $[2,11]$. For angles of incidence lower than $45^{\circ}$ resonant absorption seems to be the dominant process and to determine the temperature of the fast electron component. In contrast, for an angle of incidence around $60^{\circ}$, TPD becomes the dominant heating process for fast particles. This explains the strong temperature increase detected for an incidence of $60^{\circ}$, in agreement with the prediction of numerical simulations [2] for the dependence of the temperature of the fast electrons versus their production mechanism.

When $\theta$ exceeds $60^{\circ}$, the laser wave does not reach $n_{\mathrm{c}} / 4$ and only Raman scattering could occur. But, for $n_{\mathrm{e}}<n_{\mathrm{c}} / 4$ : it is convective [12] and it creates plasma wave fields much weaker than at $n_{\mathrm{c}} / 4$; few fast electrons are produced and their temperature is low [13].

A value of the energy contained in fast ions has been deduced from the ion collector signals although they can give only rough measures. These ion collectors were protected from secondary emission. In order to determine the energy in the fast ions, two procedures were taken for the interpretation of the charge collector signals :(1) the fastest particles were considered as hydrogen ions and the remaining of the fast particles were considered as hydrogenic carbon ions ; (2) all fast ions were considered as a mixture of two hydrogen plus one carbon ions. Taking into account this fact, the uncertainty in the estimate of energy in fast ions lay between 10 to $25 \%$ of the total energy in fast electrons. As a result of this, most of the energy in the fast electrons was lost by slowing down while entering the solid.

The occurrence of TPD has been already obtained in a similar experiment by observing $3 / 2 \omega_{0}$ harmonic [14]. The experiment had been made with the same experimental apparatus and the same irradiance. 


\section{Experiment at $0.53 \mu \mathrm{m}$.}

Comparable experiments have been made at $0.53 \mu \mathrm{m}$ for irradiances in the range of 1 to $2 \times 10^{14} \mathrm{~W} / \mathrm{cm}^{2}$. The focal spot diameter was $200 \mu \mathrm{m}$.

The low temperature value was $400 \mathrm{eV}$ at normal incidence and $300 \mathrm{eV}$ at an angle of $60^{\circ}$. As a difference with the $\mathrm{CO}_{2}$ experimental case, the X-ray signals from $1 \mathrm{keV}$ to $10 \mathrm{keV}$ showed a very similar intensity dependence versus angle of incidence (Fig. 1a). One difference from the $\mathrm{CO}_{2}$ case was the angular positions of the maximum, which is obtained at an angle of $\left(18 \pm 2^{\circ}\right)$ for the $0.53 \mu \mathrm{m}$ experiment. Moreover, a slight difference was detected in the behaviour of the high energy $X$-rays (between 10 and $30 \mathrm{keV}$ : Fig. 1 b) where we noticed a small plateau around $50^{\circ}$ of incidence and a sharp decrease beyond $60^{\circ}$ where no signal could be detected. The measured hot electron temperature $T_{\mathrm{h}}$ was $(2.5 \pm 0.5) \mathrm{keV}$. No dependence on the angle of incidence was noticed.

Figure 3 gives the variations of energy in fast electrons versus the angle of incidence. One can see that at maximum for normal incidence, $10 \%$ of the incident energy is found in the fast electrons. As for the $\mathrm{CO}_{2}$ case, the energy in the fast ions was estimated to be $10 \%$ to $20 \%$ of the fast electron energy. Around $55^{\circ}$, a level of $1 \%$ of the laser energy is still found in the fast electrons, as for the $\mathrm{CO}_{2}$ case. However, in the short wavelength irradiation, a plateau was seen in the amount in fast electron production. But no significant change in $T_{\mathrm{h}}$ could be deduced, owing to the experimental precision.

In addition to these results, the presence of the $3 / 2 \omega_{0}$ harmonic, which we attributed to the occurrence of TPD as generally assumed, has been detected. Its dependence on the angle of incidence has shown the occurrence of a maximum at an angle of $\left(50^{\circ}-60^{\circ}\right)$. Correlation of this fact with the above-mentioned results could confirm as a first interpretation that some of the fast electrons detected in the same conditions should be attributed to the TPD instability. However this production does not dominate the energetic electron generation as in the $\mathrm{CO}_{2}$ laser irradiation case at angle of $60^{\circ}$. This is probably a consequence of the effect of collisions for which in the experiment at $0.53 \mu \mathrm{m}$, the frequency is comparable to the growth rate of TPD instability. It would explain that $T_{\mathrm{h}}$ does not show a measurable increase in those conditions.

\section{Conclusion.}

In conclusion, this experiment has shown that resonant absorption and TPD are the major mechanisms for fast electron generation in laser irradiated targets and for different laser wavelengths, $10.6 \mu \mathrm{m}$ and $0.53 \mu \mathrm{m}$. The angular dependence of the fast electron generation with respect to normal target showed that, for normal and low angles of incidence, resonant absorption seems to be the dominant mechanism at the irradiances presently involved $\left(I \lambda_{0}^{2} \simeq 10^{14} \mathrm{~W} \mu \mathrm{m}^{2} /\right.$ $\mathrm{cm}^{2}$ ). For large oblique incidence, this mechanism is weakened and we then obtained evidence of a fast electron generation associated with TPD.

In the case of the long wavelength laser, there was a significant increase in the temperature of fast electrons generated by TPD compared to those associated with resonant absorption. The behaviour in this case is in agreement with the fact that TPD produces fast electrons hotter than those produced by resonant absorption [12]. The behaviour for the shorter wavelength $0.53 \mu \mathrm{m}$ is somewhat more intriguing. If more fast electrons associated with TPD were detected for oblique incidence, there was no obvious increase of their temperature. At present we have no clear explanation for this behaviour.

However, fast electrons generated by TPD under the best circumstances for their occurrence at oblique incidence represented only a tenth of the fast electrons generated by resonant absorption. Moreover, for the short wavelength case, most of the absorption is due to Inverse Bremsstrahlung, $60 \%$ of the incident energy [7] in the usual conditions of irradiation at normal or slightly oblique incidence. The fast electrons associated with TPD should represent a fraction of energy less than 
$1 \%$ and have a temperature of the order of few $\mathrm{keV}$ which could be thermalized in the dense plasma and reduce the preheating effect.

This work has been done in rather inhomogeneous plasma. Work is in progress in order to study the fast electron generation in more homogeneous plasmas, where instabilities such as TPD and Raman scattering could occur more easily.

\section{Acknowledgments.}

We thank the French D.R.E.T. (Direction des Recherches et Etudes Techniques) for financial support of this work.

\section{References}

[1] LiU, C. S., Rosenbluth, M. N., Phys. Fluids 19 (1976) 967.

Pellat, R., Parametric Instabilities, Summer School - Les Houches (France), July 1980.

[2] Langdon, A. B., Lasinski, B. F., Kruer, W. L., Phys. Rev. Lett. 43 (1979) 133.

[3] Estabrook, K., Kruer, W. L., Lasinski, B. F., Phys. Rev. Lett. 45 (1980) 1399.

[4] Loth, C., Bruneau, D., Fabre, E., Appl. Opt. 19 (1980) 1022.

[5] Pípin, H., Martin, F., Grek, B., Johnston, T. W., Kieffer, J. C., Mitchel, G., J. Appl. Phys. 50 (1979) 6784.

[6] Brueckner, K. A., Nucl. Fusion 17 (1977) 1257.

[7] Garban-Labaune, C., Fabre, E., David, F., Maignan, J., Michard, A., J. Physique-Lett. 41 (1980) L-463.

Amiranoff, F., Benattar, R., Bonnaud, G., Fabbro, R., Fabre, E., Garban-Labaune, C., Grandjouan, N., Popovics, C., Poquerusse, A., Stenz, C., Virmont, J., Weinfeld, M., Proceedings of the Japan - U.S. Seminar, Nara (Japan), May 3-7, 1982.

[8] David, F., Pellat, R., Phys. Fluids 23 (1980) 1682.

[9] Ginzburg, V. L., The Propagation of Electromagnetic Waves (Pergamon, London) 1965, Chaps. 4 and 5.

[10] Schuss, J., Phys. Fluids 20 (1977) 1121.

[11] EstabrooK, K., Kruer, W. L., Phys. Rev. Lett. 40 (1978) 42.

[12] Kaw, P. K., Kruer, W. L., Liu, C. S., Nishikawa, K., Adv. Plasma Phys. 6 (1976).

[13] Estabrook, K., Kruer, W. L., Lasinski, B. F., Phys. Rev. Lett. 45 (1980) 1399.

[14] Garban-Labaune, C., Fabre, E., Stenz, C., Popovics, C., Virmont, J., Amiranoff, F., J. PhysiqueLett. 39 (1978) L-165. 